\title{
Improved convergence analysis of the Secant method using restricted convergence domains with real-world applications
}

Ioannis K. Argyros ${ }^{a}$, Alberto Magreñán ${ }^{b, *}$, Íñigo Sarría ${ }^{b}$, Juan Antonio Sicilia ${ }^{b}$

${ }^{a}$ Cameron University, Department of Mathematics Sciences Lawton, OK 73505, USA.

${ }^{b}$ Escuela Superior de Ingeniería y Tecnología, Universidad Internacional de La Rioja, Av de la Paz, 137, 26006 Logroño, La Rioja, Spain.

\begin{abstract}
In this paper, we are concerned with the problem of approximating a solution of a nonlinear equations by means of using the Secant method. We present a new semilocal convergence analysis for Secant method using restricted convergence domains. According to this idea we find a more precise domain where the inverses of the operators involved exist than in earlier studies. This way we obtain smaller Lipschitz constants leading to more precise majorizing sequences. Our convergence criteria are weaker and the error bounds are more precise than in earlier studies. Under the same computational cost on the parameters involved our analysis includes the computation of the bounds on the limit points of the majorizing sequences involved. Different real-world applications are also presented to illustrate the theoretical results obtained in this study.
\end{abstract}

Keywords: Secant method, Banach space, majorizing sequence, divided difference, local convergence, semilocal convergence. 2010 MSC: 65H10, 65G99, 65B05, 65N30, 47H17, 49M15.

(c)2018 All rights reserved.

\section{Introduction}

In this study, we are concerned with the problem of approximating a locally unique solution $x^{\star}$ of the nonlinear equation

$$
\mathrm{F}(\mathrm{x})=0,
$$

where, $\mathrm{F}$ is a Fréchet-differentiable operator defined on a non-empty subset $\mathcal{D}$ of a Banach space $X$ with values in a Banach space $y$. Several problems from Applied Sciences including Engineering can be expressed in a form like equation (1.1) using mathematical modeling [2-6, 12-14, 16, 18-24, 28, 30]. The solutions of these equations can be found in closed form only in special cases. That is why the most solution methods for these equations are iterative.

\footnotetext{
*Corresponding author

Email addresses: iargyros@cameron.edu (Ioannis K. Argyros), alberto.magrenan@unir.net (Alberto Magreñán), inigo.sarria@unir.net (Íñigo Sarría), juanantonio.sicilia@unir.net (Juan Antonio Sicilia)
}

doi: $10.22436 /$ jnsa.011.11.01

Received: 2018-03-23 Revised: 2018-06-15 Accepted: 2018-06-19 
In this paper, we consider the convergence of the Secant method defined as

$$
x_{n+1}=x_{n}-\mathcal{A}_{n}^{-1} F\left(x_{n}\right), \mathcal{A}_{n}=\delta \mathrm{F}\left(x_{n}, x_{n-1}\right) \text { for each } n=1,2, \ldots,
$$

where $x_{-1}, x_{0}$ are initial points. Here $\mathcal{A}_{n} \in \mathcal{L}(X, y)$ is an approximation of the Fréchet-derivative $F^{\prime}$ of $\mathrm{F}$ and $\mathcal{L}(X, Y)$ stands for the space of bounded linear operators from $X$ into $y$. There is a plethora of sufficient convergence criteria for the Secant method (1.2) under Lipschitz-type conditions (1.2) (see [1-28]). It is interesting to notice that although we use very general majorizing sequences for $\left\{x_{n}\right\}$ our technique leads in the semilocal case to: weaker sufficient convergence criteria; more precise estimates on the distances $\left\|x_{n}-x_{n-1}\right\|,\left\|x_{n}-x^{*}\right\|$ and an at least as precise information on the location of the solution $x^{*}$ in many interesting special cases such as Newton's method or the Secant method.

The rest of the paper is organized as follows. In Section 2, we study the convergence of the majorizing sequences for $\left\{x_{n}\right\}$ involved in the Secant method. In Section 3, we present the semilocal convergence analysis for $\left\{x_{n}\right\}$. Finally, numerical examples are presented in the concluding Section 4.

\section{Majorizing sequences for the Secant method}

In this Section, we shall first study some scalar sequences which are related to the Secant method. by

Let there be parameters $c \geqslant 0, v \geqslant 0, k>0, k_{0}>0, k_{1}>0$, and $k_{2} \geqslant 0$. Define the scalar sequence $\left\{\alpha_{n}\right\}$

$$
\left\{\begin{array}{l}
\alpha_{-1}=0, \alpha_{0}=c, \alpha_{1}=c+v, \\
\alpha_{n+2}=\alpha_{n+1}+\frac{k_{1}\left(\alpha_{n+1}-\alpha_{n}\right)+k_{2}\left(\alpha_{n}-\alpha_{n-1}\right)\left(\alpha_{n+1}-\alpha_{n}\right)}{1-\left[k_{0}\left(\alpha_{n+1}-c\right)+k \alpha_{n}\right]} \text { for each } n=0,1,2, \ldots
\end{array}\right.
$$

Special cases of the sequence $\left\{\alpha_{n}\right\}$ have been used as majorizing sequences for Secant method by several authors. For example: Case 1 (Secant method) $k_{0}=k$ and $k_{1}=k_{2}$ has been studied in $[2,5,6,8,12,14$, $15,17,20,22-26,28-30$ ] and for $k_{0}=k, k_{1}=k_{2}$ and $k_{0} \leqslant k_{1}$ in [9-11]. Case 2 (Newton's method) $k_{2}=0$, $k=0, c=0$ and $k_{0}=k_{1}$ has been studied in $[2,5,6,8,10,13,15,17-20,22-25,27,28]$ and for $k_{0} \leqslant k_{1}$ in [2-4].

In the present paper, we shall study the convergence of sequence $\left\{\alpha_{n}\right\}$ by first simplifying it. Indeed, the purpose of the following transformations is to study the sequence (2.1) after using easier to study sequences defined by (2.3), (2.4), and (2.5). Let

$$
\lambda=\frac{k_{2}}{k_{1}}, \quad L_{0}=\frac{1}{1+k_{0} c}, \text { and } L=\frac{k_{1}}{1+k_{0} c} .
$$

Using (2.1) and (2.2), sequence $\left\{\alpha_{n}\right\}$ can be written as

$$
\left\{\begin{array}{l}
\alpha_{-1}=0, \alpha_{0}=c, \alpha_{1}=c+v, \\
\alpha_{n+2}=\alpha_{n+1}+\frac{L\left(\alpha_{n+1}-\alpha_{n}+\lambda\left(\alpha_{n}-\alpha_{n-1}\right)\right)\left(\alpha_{n+1}-\alpha_{n}\right)}{1-L_{0}\left(k_{0} \alpha_{n+1}+k \alpha_{n}\right)} \text { for each } n=0,1,2, \ldots
\end{array}\right.
$$

Notice that

$$
\mathrm{L}=\mathrm{k}_{1} \mathrm{~L}_{0} \quad \text { and } \quad \beta_{\mathrm{n}}=\mathrm{L}_{0} \alpha_{\mathrm{n}} .
$$

Then, we can define sequence $\left\{\beta_{n}\right\}$ by

$$
\left\{\begin{array}{l}
\beta_{-1}=0, \beta_{0}=L_{0} c, \beta_{1}=L_{0}(c+v) \\
\beta_{n+2}=\beta_{n+1}+\frac{k_{1}\left(\beta_{n+1}-\beta_{n}+\lambda\left(\beta_{n}-\beta_{n-1}\right)\right)\left(\beta_{n+1}-\beta_{n}\right)}{1-\left(k_{0} \beta_{n+1}+k \beta_{n}\right)} \text { for each } n=0,1,2, \ldots
\end{array}\right.
$$

Furthermore, let

$$
\gamma_{n}=\frac{1}{k_{0}+k}-\beta_{n} \text { for each } n=0,1,2, \ldots
$$


Then, sequence $\left\{\gamma_{n}\right\}$ is defined by

$$
\left\{\begin{array}{l}
\gamma_{-1}=\frac{1}{k_{0}+k}, \gamma_{0}=\frac{1}{k_{0}+k}-L_{0} c, \gamma_{1}=\frac{1}{k_{0}+k}-L_{0}(c+v), \\
\gamma_{n+2}=\gamma_{n+1}-\frac{k_{1}\left(\gamma_{n+1}-\gamma_{n}+\lambda\left(\gamma_{n}-\gamma_{n-1}\right)\right)\left(\gamma_{n+1}-\gamma_{n}\right)}{k_{0} \gamma_{n+1}+k \gamma_{n}} \text { for each } n=0,1,2, \ldots
\end{array}\right.
$$

Finally, let

$$
\delta_{n}=1-\frac{\gamma_{n}}{\gamma_{n-1}} \text { for each } n=0,1,2, \ldots
$$

Then, we define the sequence $\left\{\delta_{n}\right\}$ by

$$
\left\{\begin{array}{l}
\delta_{0}=1-\frac{\gamma_{0}}{\gamma_{-1}}, \delta_{1}=1-\frac{\gamma_{1}}{\gamma_{0}}, \\
\delta_{n+2}=\frac{k_{1} \delta_{n+1}\left(\lambda \delta_{n}+\left(1-\delta_{n}\right) \delta_{n+1}\right)}{\left(1-\delta_{n}\right)\left(1-\delta_{n+1}\right)\left(k_{0}\left(1-\delta_{n+1}\right)+k\right)} \text { for each } n=0,1,2, \ldots
\end{array}\right.
$$

It is convenient for the study of the convergence of the sequence $\left\{\alpha_{n}\right\}$ to define polynomial $p$ by

$$
p(t)=k_{0} t^{3}-\left(k_{1}+3 k_{0}+k\right) t^{2}+\left(2 k+3 k_{0}+k_{1}(\lambda+1)\right) t-\left(k_{0}+k\right) .
$$

We have that $p(0)=-\left(k_{0}+k\right)<0$ and $p(1)=k_{1} \lambda$ for $\lambda>0$. It follows from the intermediate value theorem that $p$ has roots in $(0,1)$. Denote the smallest root by $\delta$. If $\lambda=0$, then $p(t)=(t-1)\left(k_{0} t^{2}-\left(k_{1}+k+\right.\right.$ $\left.\left.2 k_{0}\right) t+k_{0}+k\right)$. Hence, we can choose the smallest root of $p$ given by $\frac{2 k_{0}+k_{1}+k-\sqrt{\left(2 k_{0}+k_{1}+k\right)^{2}-4 k_{0}\left(k_{0}+k\right)}}{2 k_{0}} \in$ $(0,1)$ to be $\delta$ in this case.

Notice also that

$$
p(t) \leqslant 0 \text { for each } t \in(-\infty, \delta] .
$$

Next, we study the convergence of these sequences starting from $\left\{\delta_{n}\right\}$.

Lemma 2.1 ([10]). Let $\delta_{1}>0, \delta_{2}>0$ and $k_{1}>0$ be given parameters. Suppose that

$$
0<\delta_{2} \leqslant \delta_{1} \leqslant \delta,
$$

where $\delta$ was defined in (2.7). Let $\left\{\delta_{n}\right\}$ be the scalar sequence defined by (2.6). Then, the following assertions hold: $\left(\mathcal{A}_{1}\right)$ If

then

$$
\delta_{1}=\delta_{2}
$$

$$
\delta_{n}=\delta \text { for each } n=1,2,3, \ldots
$$

$\left(\mathcal{A}_{2}\right)$ If

$$
\delta_{2}<\delta_{1}<\delta
$$

then sequence $\left\{\delta_{n}\right\}$ is decreasing and converges to 0 .

Lemma 2.2 ([10]). Suppose that the hypothesis (2.10) is satisfied. Then, the sequence $\left\{\gamma_{n}\right\}$ is decreasingly convergent and sequences $\left\{\alpha_{n}\right\}$ and $\left\{\beta_{n}\right\}$ are increasingly convergent.

Lemma 2.3 ([10]). Suppose that (2.8) and (2.9) hold. Then, the following assertions hold for each $\mathrm{n}=1,2, \ldots$

$$
\begin{aligned}
& \delta_{n}=\delta, \\
& \gamma_{n}=(1-\delta)^{n} \gamma_{0}, \gamma^{*}=\lim _{n \rightarrow \infty} \gamma_{n}=0, \\
& \beta_{n}=\frac{1}{k_{0}+k}-(1-\delta)^{n} \gamma_{0}, \beta^{*}=\lim _{n \rightarrow \infty} \beta_{n}=\frac{1}{k_{0}+k^{\prime}},
\end{aligned}
$$

and

$$
\alpha_{n}=\frac{1}{L_{0}}\left[\frac{1}{k_{0}+k}-(1-\delta)^{n} \gamma_{0}\right], \alpha^{*}=\lim _{n \rightarrow \infty} \alpha_{n}=\frac{1}{L_{0}\left(k_{0}+k\right)}
$$


Corollary 2.4 ([10]). Suppose that the hypotheses of Lemma 2.1 and Lemma 2.2 hold. Then, sequence $\left\{\alpha_{n}\right\}$ defined in (2.1) is nondecreasing and converges to

$$
\alpha^{*}=\beta^{*}\left(1+k_{0} c\right) .
$$

Next, we present lower and upper bounds on the limit point $\alpha^{*}$.

Lemma 2.5 ([10]). Suppose that condition (2.10) is satisfied. Then, the following assertion holds:

$$
b_{1}^{1} \leqslant \alpha^{*} \leqslant b_{2}^{1}
$$

where

$$
\begin{aligned}
& \mathrm{b}_{1}^{1}=\frac{1+\mathrm{k}_{0} \mathrm{c}}{1-\mathrm{kc}}\left[\frac{1}{\mathrm{k}_{0}+\mathrm{k}}-\exp \left(-2\left(\frac{\delta_{1}}{2-\delta_{1}}+\frac{\delta_{2}}{2-\delta_{2}}\right)\right)\right], \\
& \mathrm{b}_{2}^{1}=\frac{1+\mathrm{k}_{0} \mathrm{c}}{1-\mathrm{kc}}\left[\frac{1}{\mathrm{k}_{0}+\mathrm{k}}-\exp \left(\delta^{*}\right)\right], \\
& \delta^{*}=-\left[\frac{1}{1-\delta_{1}}\left(\delta_{1}+\frac{\delta_{2}}{1-\mathrm{r}}\right)+\ln \left(\frac{\left(\mathrm{k}_{0}+\mathrm{k}\right)\left(1+\mathrm{k}_{0} \mathrm{c}\right)}{1-\mathrm{kc}}\right)\right],
\end{aligned}
$$

and

$$
r=k_{1} \frac{\lambda \delta_{1}+\delta_{2}\left(1-\delta_{1}\right)}{\left(1-\delta_{1}\right)\left(1-\delta_{2}\right)\left(k+k_{0}\left(1-\delta_{2}\right)\right)}
$$

From now on we shall denote by $\left(\mathrm{C}^{1}\right)$ the hypotheses of Lemma 2.1 and Lemma 2.2. Remark 2.6.

(a) Let us introduce the notation

$$
c^{N}=\alpha_{N-1}-\alpha_{N-2}, v^{N}=\alpha_{N}-\alpha_{N-1}
$$

for some integer $N \geqslant 1$. Notice that $c^{1}=\alpha_{0}-\alpha_{-1}=c$ and $v^{1}=\alpha_{1}-\alpha_{0}=v$. The results in the preceding Lemmas can be weakened even further as follows. Consider the convergence criteria $\left(C_{*}^{N}\right)$ for $N>1:\left(C^{1}\right)$ with $c, v$ replaced by $c^{N}, v^{N}$, respectively

$$
\alpha_{-1}<\alpha_{0}<\alpha_{1}<\cdots<\alpha_{N}<\alpha_{N+1}, \quad k_{0}\left(\alpha_{N+1}-c^{N}\right)+k \alpha_{N}<1 .
$$

Then, the preceding results hold with $c, v, \delta_{1}, \delta_{2}, b_{1}^{1}, b_{2}^{1}$ replaced, respectively by $c^{N}, v^{N}, \delta_{N}, \delta_{N+1}, b_{1}^{N}, b_{2}^{N}$. (b) Notice that if

$$
k_{0}\left(\alpha_{N+1}-c^{N}\right)+k \alpha_{N}<1 \text { holds for each } n=0,1,2, \ldots,
$$

then, it follows from (2.1) that sequence $\left\{\alpha_{n}\right\}$ is increasing, bounded from above by $\frac{1+k_{0} c}{k_{0}+k}$ and as such it converges to its unique least upper bound $\alpha^{*}$. Criterion (2.11) is the weakest of all the preceding convergence criteria for sequence $\left\{\alpha_{n}\right\}$. Clearly all the preceding criteria imply (2.11). Finally, define the criteria for $N \geqslant 1$

$$
\left(\mathrm{I}^{\mathrm{N}}\right)=\left\{\begin{array}{l}
\left(\mathrm{C}_{*}^{\mathrm{N}}\right), \\
(2.11) \text { if criteria }\left(\mathrm{C}_{*}^{\mathrm{N}}\right) \text { fails. }
\end{array}\right.
$$

Lemma 2.7 ([10]). Suppose that the conditions (2.10) hold. Then, the following assertion holds

$$
\mathrm{b}_{1}^{1} \leqslant \alpha^{*} \leqslant \mathrm{~b}_{2}^{1},
$$

where

$$
\mathrm{b}_{1}^{1}=\frac{1+\mathrm{k}_{0} \mathrm{c}}{1-\mathrm{kc}}\left[\frac{1}{\mathrm{k}_{0}+\mathrm{k}}-\exp \left(-2\left(\frac{\delta_{1}}{2-\delta_{1}}+\frac{\delta_{2}}{2-\delta_{2}}\right)\right)\right]
$$




$$
\begin{aligned}
& \mathrm{b}_{2}^{1}=\frac{1+\mathrm{k}_{0} \mathrm{c}}{1-\mathrm{kc}}\left[\frac{1}{\mathrm{k}_{0}+\mathrm{k}}-\exp \left(\delta^{*}\right)\right] \\
& \delta^{*}=-\left[\frac{1}{1-\delta_{1}}\left(\delta_{1}+\frac{\delta_{2}}{1-\mathrm{r}}\right)+\ln \left(\frac{\left(\mathrm{k}_{0}+\mathrm{k}\right)\left(1+\mathrm{k}_{0} \mathrm{c}\right)}{1-\mathrm{kc}}\right)\right],
\end{aligned}
$$

and

$$
r=k_{1} \frac{\lambda \delta_{1}+\delta_{2}\left(1-\delta_{1}\right)}{\left(1-\delta_{1}\right)\left(1-\delta_{2}\right)\left(k+k_{0}\left(1-\delta_{2}\right)\right)}
$$

\section{Semilocal convergence of the Secant method}

In this section, we first present the semilocal convergence of the Secant method using $\left\{\alpha_{n}\right\}$ (defined in (2.1)) as a majorizing sequence. Let $U(x, R)$ stand for an open ball centered at $x \in X$ with radius $R>0$. Let $\bar{U}(x, R)$ denote its closure. We shall study the Secant method for triplets $\left(\mathcal{F}, x_{-1}, x_{0}\right)$ belonging to the class $\mathcal{K}=\mathcal{K}\left(v, c, k, k_{0}, k_{1}, k_{2}\right)$ defined as follows.

Definition 3.1. Let $v, c, k, k_{0}, k_{1}, k_{2}$ be constants satisfying the hypotheses $\left(I^{N}\right)$ for some fixed integer $N \geqslant 1$. A triplet $\left(\mathcal{F}, x_{-1}, x_{0}\right)$ belongs to the class $\mathcal{K}=\mathcal{K}\left(v, c, k_{,} k_{0}, k_{1}, k_{2}\right)$ if:

$\left(\mathcal{D}_{1}\right) \mathcal{F}$ is a nonlinear operator defined on a convex subset $D$ of a Banach space $X$ with values in a Banach space $y$;

$\left(\mathcal{D}_{2}\right) x_{-1}$ and $x_{0}$ are two points belonging to the interior $\mathrm{D}^{0}$ of $\mathrm{D}$ and satisfying the inequality

$$
\left\|x_{0}-x_{-1}\right\| \leqslant c,
$$

for some constant $c \geqslant 0$;

$\left(\mathcal{D}_{3}\right) \mathcal{F}$ is Fréchet-differentiable on $\mathrm{D}^{0}$ and there exists an operator $\delta \mathcal{F}: \mathcal{D}^{0} \times \mathrm{D}^{0} \rightarrow \mathrm{E}(\mathrm{X}, \mathrm{Y})$ such that $\delta \mathcal{F}(x, y)(x-y)=F(x)-F(y)$ for each $x \neq y, \delta \mathcal{F}(x, x)=F^{\prime}(x), x \in D^{0}, F^{\prime}\left(x_{0}\right)^{-1}, \mathcal{A}^{-1}=\delta \mathcal{F}\left(x_{0}, x_{-1}\right)^{-1} \in$ $€(Y, X)$ for all $x, y \in D$ then, the following hold

$$
\left\|\mathcal{A}^{-1} \mathcal{F}\left(x_{0}\right)\right\| \leqslant v, \quad\left\|F^{\prime}\left(x_{0}\right)^{-1}\left(\delta \mathcal{F}(x, y)-F^{\prime}\left(x_{0}\right)\right)\right\| \leqslant k_{0}\left\|x-x_{0}\right\|+k\left\|y-x_{0}\right\|
$$

and for each $x, y, z \in D_{0}:=U\left(x_{0}, \frac{1}{k_{0}+k}\right) \cap D$

$$
\left\|F^{\prime}\left(x_{0}\right)^{-1}\left(\delta \mathcal{F}(x, y)-F^{\prime}(z)\right)\right\| \leqslant k_{1}\|x-z\|+k_{2}\|y-z\|
$$

for some constants $k>0, k_{0}>0, k_{1}>0, k_{2} \geqslant 0$ and $v \geqslant 0$;

$\left(\mathcal{D}_{4}\right)$

$$
\overline{\mathrm{U}}\left(\mathrm{x}_{0}, \alpha^{*}-\mathrm{c}\right) \subseteq \mathrm{D} \text { or } \mathrm{U}\left(\mathrm{x}_{0}, \frac{1}{\mathrm{k}_{0}+\mathrm{k}}\right) \subset \mathrm{D},
$$

where $\alpha^{*}$ is given in Lemma 2.3.

Next, we present the semilocal convergence result for the Secant method.

Theorem 3.2. If $\left(\mathcal{F}, x_{-1}, x_{0}\right) \in \mathcal{K}\left(v, c, k, k_{0}, k_{1}, k_{2}\right)$, then the sequence $\left\{x_{n}\right\}(n \geqslant-1)$ generated by the Secant method is well defined, remains in $\overline{\mathrm{U}}\left(\mathrm{x}_{0}, \alpha_{0}^{*}\right)$ for each $\mathrm{n}=0,1,2, \ldots$ and converges to a unique solution $x^{*} \in$ $\overline{\mathrm{u}}\left(\mathrm{x}_{0}, \alpha^{*}-\mathrm{c}\right)$ of (1.1). Moreover, the following assertions hold for each $\mathrm{n}=0,1,2, \ldots$

$$
\left\|x_{n}-x_{n-1}\right\| \leqslant \alpha_{n}-\alpha_{n-1}
$$

and

$$
\left\|x^{*}-x_{n}\right\| \leqslant \alpha^{*}-\alpha_{n},
$$

where sequence $\left\{\alpha_{n}\right\}(n \geqslant 0)$ is given in (2.1). Furthermore, if there exists $R$ such that

$$
\overline{\mathrm{U}}\left(\mathrm{x}_{0}, \mathrm{R}\right) \subseteq \mathrm{D}, \mathrm{R} \geqslant \alpha^{*}-\mathrm{c} \text { and } \mathrm{k}_{0}\left(\alpha^{*}-\alpha_{0}\right)+\mathrm{kR}<1,
$$

then, the solution $x^{*}$ is unique in $\overline{\mathrm{U}}\left(\mathrm{x}_{0}, \mathrm{R}\right)$. 
Proof. Simply notice that the iterates lie in $\mathrm{D}_{0}$ which is a more accurate location containing the iterates $\left\{x_{n}\right\}$ than D used in [10], since $D_{0} \subseteq \mathrm{D}$. Then, the proof is exactly the same with the one in [10].

Remark 3.3. If follows from the proof of Theorem 3.2 that sequence $\left\{r_{n}\right\}$ defined by

$$
\left\{\begin{array}{l}
r_{-1}=0, r_{0}=c, r_{1}=c+v, \\
r_{2}=r_{1}+\frac{\left(k_{0}\left(r_{1}-r_{0}\right)+k\left(r_{0}-r_{-1}\right)\left(r_{1}-r_{0}\right)\right.}{1-\left(k_{0}\left(r_{1}-c\right)+k c\right)}, \\
r_{n+2}=r_{n+1}+\frac{\left(k_{1}\left(r_{n+1}-r_{n}\right)+k_{2}\left(r_{n}-r_{n-1}\right)\right)\left(r_{n+1}-r_{n}\right)}{1-\left(k_{0}\left(r_{n+1}-c\right)+k r_{n}\right)}
\end{array}\right.
$$

is a more precise majorizing sequences for $\left\{x_{n}\right\}$. Clearly, the sequence $\left\{r_{n}\right\}$ also converges under the $\left(I^{N}\right)$ hypotheses.

A simple inductive argument shows that if $k_{0}<k_{1}$ or $k<k_{2}$ for each $n=2,3, \ldots$,

$$
\begin{gathered}
r_{n}<\alpha_{n}, \\
r_{n+1}-r_{n}<\alpha_{n+1}-\alpha_{n},
\end{gathered}
$$

and

$$
r^{*}=\lim _{n \rightarrow \infty} r_{n} \leqslant \alpha^{*}=\lim _{n \rightarrow \infty} \alpha_{n} .
$$

Note also that sequence $\left\{r_{n}\right\}$ may converge under even weaker hypotheses. The sufficient convergence criterion (2.12) determines the smallness of $c$ and $r$. This criterion can be solved for $c$ and $r$ (see for example the $h$ criteria or (3.5) in the following). Indeed, let us demonstrate the advantages in two popular cases:

Case 1: Newton's method (i.e., if $c=0, k_{0}=k, k_{1}=k_{2}$ ). Then, it can easily be seen that $\left\{s_{n}\right\}$ (and consequently $\left\{r_{n}\right\}$ ) converges provided that (see also [8])

$$
h_{2}=\xi_{2} v \leqslant 1 \text {, }
$$

where

$$
\xi_{2}=\frac{1}{4}\left(4 k_{0}+\sqrt{k_{0} k_{1}}+\sqrt{k_{0} k_{1}+8 k_{0}^{2}}\right),
$$

whereas sequence $\left\{x_{n}\right\}$ converges, if

$$
h_{1}=k_{1} v \leqslant 1,
$$

where

$$
\xi_{1}=\frac{1}{4}\left(4 k_{0}+k_{1}+\sqrt{k_{0}^{2}+8 k_{1} k_{0}}\right) .
$$

In the case $k_{0}=k_{1}$, we obtain the famous for its simplicity and clarity Kantorovich sufficient convergent criteria $[5,20]$ given by

$$
h=2 k_{1} v \leqslant 1 \text {. }
$$

Notice however that

$$
\mathrm{h} \leqslant 1 \Rightarrow \mathrm{h}_{1} \leqslant 1 \Rightarrow \mathrm{h}_{2} \leqslant 1
$$

but not necessarily vice versa unless if $k_{0}=k_{1}$. Moreover, we have that

$$
\frac{h_{1}}{h} \rightarrow \frac{1}{4}, \frac{h_{1}}{h} \rightarrow 0, \frac{h_{2}}{h_{1}} \rightarrow 0 \text { as } \frac{k_{0}}{k_{1}} \rightarrow 0 .
$$

Case 2: Secant method. Schmidt [27], Potra-Ptáck [24], Dennis [14], and Ezquerro el at. [15, 18], used the majorizing sequence $\left\{r_{n}\right\}$ for $k_{0}=k=k_{1}=k_{2}$. That is, they used the sequence $\left\{t_{n}\right\}$ given by

$$
\left\{\begin{array}{l}
t_{-1}=0, t_{0}=c, t_{1}=c+v \\
t_{n+2}=t_{n+1}+\frac{k_{1}\left(t_{n+1}-t_{n-1}\right)\left(t_{n+1}-t_{n}\right)}{1-k_{1}\left(t_{n}-t_{n+1}+c\right)}
\end{array}\right.
$$


whereas our sequence $\left\{\alpha_{n}\right\}$ for $k_{0}=k$ and $k_{1}=k_{2}[1,5-12,15,18,25,27]$ reduces to

$$
\left\{\begin{array}{l}
\alpha_{-1}=0, \alpha_{0}=c, \alpha_{1}=c+v, \\
\alpha_{n+2}=\alpha_{n+1}+\frac{k_{1}\left(\alpha_{n+1}-\alpha_{n-1}\right)\left(\alpha_{n+1}-\alpha_{n}\right)}{1-k_{0}\left(\alpha_{n+1}-\alpha_{n}+c\right)} .
\end{array}\right.
$$

Then, in case $k_{0}<k_{1}$ our sequence is more precise (see also (3.1)-(3.3)). Notice also that in the preceding references the sufficient convergence criterion associated to $\left\{t_{n}\right\}$ is given by

$$
k_{1} c+2 \sqrt{k_{1} v} \leqslant 1
$$

Our sufficient convergence criteria are weaker in this case. It is worth nothing that if $c=0$ (3.5) reduces to (3.4). Similar observations can be made for other choices of parameters.

Finally, notice that we can obtain and use even smaller Lipschitz constants, if we simply work on $\mathrm{D}_{1}:=\mathrm{U}\left(\mathrm{x}_{1}, \frac{1}{\mathrm{k}_{0}+\mathrm{k}}-\left\|\mathrm{x}_{1}-\mathrm{x}_{0}\right\|\right)$ instead of $\mathrm{D}_{0}$, since we are still using initial data $\left(\mathrm{x}_{1}=\mathrm{x}_{0}-\mathcal{A}_{0}^{-1} \mathrm{~F}\left(\mathrm{x}_{0}\right)\right)$.

\section{Applications}

Application 4.1. Let $X=Y=\mathcal{C}[0,1]$, the space of continuous functions defined in $[0,1]$ equipped with the max-norm. Let $\Omega=\{x \in \mathcal{C}[0,1] ;\|x\| \leqslant R\}$, such that $R>1$ and $F$ defined on $\Omega$ and given by

$$
F(x)(s)=x(s)-f(s)-\lambda \int_{0}^{1} G(s, t) x(t)^{3} d t, \quad x \in C[0,1], s \in[0,1],
$$

where $f \in \mathcal{C}[0,1]$ is a given function, $\Lambda$ is a real constant and the kernel $G$ is the Green function

$$
G(s, t)= \begin{cases}(1-s) t, & t \leqslant s \\ s(1-t), & s \leqslant t\end{cases}
$$

In this case, for each $x \in \Omega, F^{\prime}(x)$ is a linear operator defined on $\Omega$ by the following expression:

$$
\left[F^{\prime}(x)(v)\right](s)=v(s)-3 \Lambda \int_{0}^{1} G(s, t) x(t)^{2} v(t) d t, \quad v \in C[0,1], s \in[0,1]
$$

If we choose $x_{0}(s)=f(s)=1, x_{-1}=0.9$, it follows $\left\|I-F^{\prime}\left(x_{0}\right)\right\| \leqslant 3|\Lambda| / 8$. Thus, if $|\Lambda|<8 / 3, F^{\prime}\left(x_{0}\right)^{-1}$ is defined and

$$
\left\|F^{\prime}\left(x_{0}\right)^{-1}\right\| \leqslant \frac{8}{8-3|\Lambda|}
$$

Moreover,

$$
\left\|\mathrm{F}\left(\mathrm{x}_{0}\right)\right\| \leqslant \frac{|\Lambda|}{8}
$$

Define the divided difference defined by

$$
\delta F(x, y)=\int_{0}^{1} F^{\prime}(y+t(x-y)) d t .
$$

Choosing $\Lambda=1$ and $R=1.5$, we have

$c=0.1, k_{0}=1, k=0.5, k_{1}=1.06666 \ldots, k_{2}=0.533333 \ldots, L_{0}=0.909091 \ldots, L=0.969697 \ldots$, and $v=0.2$.

Moreover, since $\frac{1}{\mathrm{k}_{0}+\mathrm{k}}=0.66666 \ldots$ we obtain that

$$
\mathrm{U}\left(\mathrm{x}_{0}, \frac{1}{\mathrm{k}_{0}+\mathrm{k}}\right) \subset \mathrm{D} .
$$


Then, by the definition of the $\delta_{1}, \delta_{2}$, and $\delta$ we obtain

$$
0<\delta_{2}=0.164103 \ldots \leqslant \delta_{1}=0.315789 \ldots \leqslant \delta=0.487078 \ldots
$$

So we can ensure the convergence of the Secant method to the solution of the equation. Recall that the previous conditions considered by other authors are violated since

$$
k_{1} c+2 \sqrt{k_{1} v}=1.03043 \ldots \geqslant 1
$$

and the convergence was not ensured.

Application 4.2. We consider the following Planck's radiation law problem found in [19]:

$$
\varphi(\lambda)=\frac{8 \pi c P \lambda^{-5}}{e^{\frac{c P}{\lambda B T}-1}}
$$

which calculates the energy density within an isothermal blackbody, where

- $\lambda$ is the wavelength of the radiation;

- $\mathrm{T}$ is the absolute temperature of the blackbody;

- B is Boltzmann's constant;

- P is the Planck's constant;

- $\mathrm{c}$ is the speed of light.

Suppose, we would like to determine wavelength $\lambda$ which corresponds to maximum energy density $\varphi(\lambda)$. From (4.1), we get

$$
\varphi^{\prime}(\lambda)=\left(\frac{8 \pi c P \lambda^{-6}}{e^{\frac{c P}{\lambda B T}-1}}\right)\left(\frac{\left(\frac{c P}{\lambda B T}\right) e^{\frac{c P}{\lambda B T}-1}}{e^{\frac{c P}{\lambda k T}}-1}-5\right) .
$$

The maxima for $\varphi$ occurs when

$$
\frac{\left(\frac{c P}{\lambda B T}\right) e^{\frac{{ }^{P P}}{\lambda B T}-1}}{e^{\frac{c P}{\lambda B T}-1}}=5 .
$$

Here putting $x=\frac{\mathrm{cP}}{\lambda \mathrm{BT}}$, the above equation becomes

$$
1-\frac{x}{5}=e^{-x}
$$

Let us define

$$
f(x)=e^{-x}-1+\frac{x}{5} .
$$

As a consequence, finding the roots of (4.2) gives us the maximum wavelength of radiation $(\lambda)$ by means of the following formula:

$$
\lambda \approx \frac{\mathrm{cP}}{x^{*} \mathrm{BT}} .
$$

It is easy to see that function $f(x)$ is continuous and that $f(2)=-0.464665 \ldots$ and $f(7)=0.400912 \ldots$. Then, it follows from the Intermediate Value Theorem that $f(x)$ has zeros in the interval $(2,7)$.

We consider $\mathcal{D}=[2,7]$. Then, choosing $x_{0}=4$ and Newton's method we obtain that

$$
\mathrm{k}_{0}=\mathrm{k}=0.161021 \ldots, \mathrm{k}_{1}=\mathrm{k}_{2}=0.372446 \ldots, \mathrm{L}_{0}=1, \mathrm{~L}=0.372446 \ldots, \text { and } \eta=1 \text {. }
$$


Moreover, since $\frac{1}{\mathrm{k}_{0}+\mathrm{k}}=0.0328022 \ldots$ we obtain that

$$
\mathrm{U}\left(\mathrm{x}_{0}, \frac{1}{\mathrm{k}_{0}+\mathrm{k}}\right) \subset \mathrm{D} .
$$

Then, by the definition of the $\delta_{1}, \delta_{2}$, and $\delta$ we obtain

$$
0<\delta_{2}=0.210873 \ldots \leqslant \delta_{1}=0.322041 \ldots \leqslant \delta=0.407721 \ldots
$$

So we can ensure the convergence of the Newton's method to the solution $x^{*}=4.96511 \ldots$ of $f(x)$. Recall that the previous conditions considered by other authors are violated since

$$
k_{1} c+2 \sqrt{k_{1} v}=1.22057 \ldots \geqslant 1
$$

and the convergence was not ensured.

\section{Acknowledgment}

This research was supported by Universidad Internacional de La Rioja (UNIR, http:/ /www.unir.net), under the Plan Propio de Investigación, Desarrollo e Innovación 3 [2015-2017]. Research group: MAPPING, by the the grant SENECA 19374/PI/14 and by Ministerio de Ciencia y Tecnología MTM2014-52016C2-01-P.

\section{References}

[1] S. Amat, S. Busquier, On a higher order Secant method, Appl. Math. Comput., 141 (2003), 321-329. 1, 3

[2] S. Amat, S. Busquier, M. Negra, Adaptive approximation of nonlinear operators, Numer. Funct. Anal. Optim., 25 (2004), 397-405. 1, 2

[3] S. Amat, J. A. Ezquerro, M. A. Hernández-Vern, Approximation of inverse operators by a new family of high-order iterative methods, Numer. Linear Algebra Appl., 21 (2014), 629-664.

[4] S. Amat, M. A. Hernández-Vern, M. J. Rubio, Improving the applicability of the Secant method to solve nonlinear systems of equations, Appl. Math. Comput., 247 (2014), 741-752. 2

[5] I. K. Argyros, Computational theory of iterative methods, Elsevier B. V., Amsterdam, (2007). 2, 3, 3

[6] I. K. Argyros, Y. J. Cho, S. Hilout, Numerical method for equations and its applications, CRC Press, Boca Raton, (2012). 1,2

[7] I. K. Argyros, D. González, Á. A. Magreñán, A semilocal convergence for a uniparametric family of efficient Secant-like methods, J. Funct. Spaces, 2014 (2014), 10 pages.

[8] I. K. Argyros, S. Hilout, Weaker conditions for the convergence of Newton's method, J. Complexity, 28 (2012), $364-387$. 2,3

[9] I. K. Argyros, Á. A. Magreñán, A unified convergence analysis for secant-type methods, J. Korean Math. Soc., 51 (2014), 1155-1175. 2

[10] I. K. Argyros, Á. A. Magreñán, Expanding the applicability of the secant method under weaker conditions, Appl. Math. Comput., 266 (2015), 1000-1012. 2, 2.1, 2.2, 2.3, 2.4, 2.5, 2.7, 3

[11] I. K. Argyros, Á. A. Magreñán, Relaxed Secant-type methods, Nonlinear Stud., 21 (2014), 485-503. 2

[12] E. Cătinaş, The inexact, inexact perturbed, and quasi-Newton methods are equivalent models, Math. Comp., 74 (2005), 291-301. 1, 2, 3

[13] S. Chandrasekhar, Radiative transfer, Dover Publ., New York, (1960). 2

[14] J. E. Dennis, Toward a unified convergence theory for Newton-like methods, Nonlinear Functional Anal. and Appl., 1970 (1970), 425-472. 1, 2, 3

[15] J. A. Ezquerro, J. M. Gutiérrez, M. A. Hernández, N. Romero, M. J. Rubio, The Newton method: from Newton to Kantorovich (Spanish), Gac. R. Soc. Mat. Esp., 13 (2010), 53-76. 2, 3

[16] N. Farhane, I. Boumhidi, J. Boumhidi, Smart Algorithms to Control a Variable Speed Wind Turbine, Int. J. Interact. Multimed. Artif. Intell., 4 (2017), 88-95. 1

[17] W. B. Gragg, R. A. Tapia, Optimal error bounds for the Newton-Kantorovich theorem, SIAM J. Numer. Anal., 11 (1974), 10-13. 2

[18] M. A. Hernández, M. J. Rubio, J. A. Ezquerro, Secant-like methods for solving nonlinear integral equations of the Hammerstein type, J. Comput. Appl. Math., 115 (2000), 245-254. 1, 3 
[19] D. Jain, Families of Newton-like methods with fourth-order convergence, Int. J. Comput. Math., 90 (2013), $1072-1082$. 4.2

[20] L. V. Kantorovich, G. P., Akilov, Functional Analysis (Translated from the Russian by Howard L. Silcock), Pergamon Press, Oxford-Elmsford, (1982). 2, 3

[21] R. Kaur, S. Arora, Nature Inspired Range Based Wireless Sensor Node Localization Algorithms, Int. J. Interact. Multimed. Artif. Intell., 4 (2017), 7-17.

[22] Á. A. Magreñán, A new tool to study real dynamics: The convergence plane, Appl. Math. Comput., 248 (2014), $215-224$. 2

[23] J. M. Ortega, W. C. Rheinboldt, Iterative Solution of Nonlinear Equations in Several Variables, Academic press, New York-London, (1970).

[24] F. A. Potra, V. Pták, Nondiscrete induction and iterative processes, Pitman (Advanced Publishing Program), Boston, (1984). 1, 3

[25] P. D. Proinov, General local convergence theory for a class of iterative processes and its applications to Newton's method, J. Complexity, 25 (2009), 38-62. 2, 3

[26] W. C. Rheinboldt, An adaptative continuation process for solving systems of nonlinear equations, Banach Ctz. Publ., 3 (1975), 129-142. 2

[27] J. W. Schmidt, Untere fehlerschranken fr regula-falsi-verfahren, Period. Hungar., 9 (1978), 241-247. 2, 3

[28] J. F. Traub, Iterative method for solutions of equations, Prentice-Hall, Englewood Cliffs, (1964). 1, 1, 2

[29] T. Yamamoto, A convergence theorem for Newton-like methods in Banach spaces, Numer. Math., 51 (1987), $545-557$.

[30] P. P. Zabrejko, D. F. Nguen, The majorant method in the theory of Newton-Kantorovich approximations and the Pták error estimates, Numer. Funct. Anal. Optim., 9 (1987), 671-684. 1, 2 